Check for updates

Cite this: RSC Adv., 2019, 9, 22577

Received 6th June 2019

Accepted 12th July 2019

DOI: 10.1039/c9ra04271g

rsc.li/rsc-advances

\section{Efficient and chromaticity-stable flexible white organic light-emitting devices based on organic- inorganic hybrid color-conversion electrodes $\uparrow$}

\author{
Cuiyun Peng, $\dot{t}^{\mathrm{a}}$ Kangping Liu, $\mathbf{t}^{\mathrm{b}}$ Shuanglong Wang, ${ }^{\mathrm{b}}$ Nanjie $\mathrm{Yu},{ }^{\mathrm{c}}$ Zhenyu Tang, ${ }^{\mathrm{b}}$ \\ Zhitian Ling, ${ }^{\mathrm{b}}$ Hong Lian, ${ }^{\mathrm{b}}$ Guo Chen, ${ }^{\text {b }}$ Yingjie Liao, ${ }^{\mathrm{b}}$ Weixia Lan ${ }^{\mathrm{a}}$ and Bin Wei (iD *a
}

\begin{abstract}
We have developed a novel organic-inorganic hybrid color conversion electrode composed of Ag NWs/poly [2-methoxy-5-(2-ethylhexyloxy)-1,4-phenylenevinylene] (MEH-PPV) via a solution process, which is the first report on a color conversion electrode for applications in flexible optoelectronics. Using the Ag NWs/MEHPPV composite film as the anode on polyethylene terephthalate substrate and combined with a blue organic light emitting devices (OLEDs) unit employing bis(3,5-difluoro-2-(2-pyridyl)phenyl-(2carboxypyridyl)iridium(III)) (Flrpic) in 1,3-bis(carbazol-9-yl)benzene (mCP) as the emitting layer, a highly efficient and chromaticity-stable color-conversion flexible white OLEDs (WOLEDs) is achieved with a maximum current efficiency of $20.5 \mathrm{~cd} \mathrm{~A}^{-1}$. To the best of our knowledge, this is the highest efficiency reported for color-conversion based flexible WOLEDs. Our work provides an approach to achieving highperformance flexible WOLEDs devices and demonstrates great potential for lighting and display applications.
\end{abstract}

\section{Introduction}

The high-efficiency organic light-emitting devices (OLEDs) have attracted significant interests for next-generation solid-state lighting and display applications. ${ }^{1-3}$ Theoretically, white OLEDs (WOLEDs) can be simply obtained by additive mixing of three primary organic emitting layers or two complementary colors. ${ }^{4,5}$ In general, the fabrication of WOLEDs usually employs a single emissive layer doped simultaneously with different color dopants, or vertical stacking of several single OLED units structures with different colors emissions. ${ }^{6}$ Although highly efficient WOLEDs have been achieved by using these methods, these WOLEDs often deteriorate from serious color shift over time due to the time-dependent EL spectra along with the varying voltages and the carrier recombination zone shift. Moreover, the luminogens doping concentration should be carefully tuned to realize a balanced exciton distribution profile among the emissive layers, which contends against the low cost requirement in general applications. ${ }^{7,8}$ These disadvantages could be remedied by combining single blue OLEDs and a color conversion layer

${ }^{a}$ School of Mechatronic Engineering and Automation, Shanghai University, 149 Yanchang Road, Shanghai, 200072, P. R. China.E-mail: bwei@shu.edu.cn

${ }^{b}$ Key Laboratory of Advanced Display and System Applications, Ministry of Education, Shanghai University, 149 Yanchang Road, Shanghai, 200072, P. R. China. E-mail: chenguo@shu.edu.cn

'Department of Electrical and Computer Engineering, University of Illinois at UrbanaChampaign, 306 N. Wright St., Urbana, Illinois 61801, USA

$\dagger$ Electronic supplementary information (ESI) available. See DOI: 10.1039/c9ra04271g

\$ These authors contributed equally.
(CCL) to simplify the fabrication process and achieve high color stability. ${ }^{9}$ In this scheme, the CCL can absorb photons originated from the blue EL and consequently the blue photons are downconverted to longer-wavelength colors, generating white light emission through mixing with the redundant and unconverted blue photons. OLED devices with a single blue emitter in this socalled down-conversion scheme are essentially monochromatic which can avoid energy transferring from various band gap emitters and color shifts that result from the complexity of mixed different colors in a single device. ${ }^{10}$

On the other hand, the high-performance conductive films are a crucial component of the flexible WOLEDs and a variety of other optoelectronic devices, as a high-performance flexible conductor will be required to enlarge these devices from the lab scale to the commercial scale. ${ }^{\mathbf{1 1}}$ The most commonly used flexible conducting materials are primarily sputtered indium tin oxide (ITO) with excellent electrical and optical properties. However, several crucial drawbacks, such as the high cost, the limited reserves of indium and the brittle nature hinder its sustainable applications in flexible electronics in the future. ${ }^{\mathbf{1 2 , 1 3}}$ With the emerging and rapid development of flexible organic electronics, great efforts have been made to develop alternative conductors with high flexibility including conducting polymers, metal nanowires, metal meshes, carbon nanotubes, graphene and even graphite. ${ }^{\mathbf{1 4 - 1 7}}$ Among them, silver nanowires (Ag NWs) film are an appealing candidate because of their superior features such as desirable transparent property, excellent flexibility and low sheet resistance. ${ }^{18}$ Very recently, our group demonstrated a high-performance all-solution-processable flexible organic solar cells based on opaque graphite bottom 
cathode and a transparent silver nanowires (Ag NWs) top anode. ${ }^{19}$ However, the performance of $\mathrm{Ag}$ NWs percolation networks is still not as good as ITO and there are still addressed disadvantages that inhibit the commercial application of $\mathrm{Ag}$ NWs as the electrodes in flexible electronics due to their high peaks created by overlapping junctions, poor adhesion between the $\mathrm{Ag}$ NWs and substrate and relatively large contact resistance. ${ }^{20}$

To overcome these drawbacks, various methods have been proposed. Gaynor et al. applied a flat-plate press to embed the nanowires into the polymer, demonstrating a solutionprocessed Ag nanowire/poly(methyl methacrylate) composite electrode with improved electrical conductivity and roughness and achieving an efficient flexible WOLEDs. ${ }^{21}$ Lian et al. developed an effective flexible conductive film for OLEDs by embedding Ag NWs into a non-conducting poly(vinylalcohol) to form highly smooth, conductive, and uniform composite electrode. ${ }^{22}$ However, the above methods require an excessively thick polymer supporting layer, which may lead to an increased driving voltage and/or electron-hole imbalance due to the low conductivity and transparency of the polymers, thus deteriorating the device performance. Although recent demonstrations use transparent conductive materials such as PEDOT:PSS or a buffer layer to overcoat the Ag NWs to reduce the surface roughness, these methods are usually complex and need additional materials, which increases the cost of the electrode. ${ }^{\mathbf{2 3 2 4}}$

In this study, we report a solution-processed Ag NWs/poly[2methoxy-5-(2-ethylhexyloxy)-1,4-phenylenevinylene] (MEH-PPV) color conversion electrode for high-efficiency flexible WOLEDs. An efficient hot-pressing method is utilized to improve the conductivity and smoothness of the Ag NWs/MEHPPV composite film. The parameters of hot-pressing are carefully adjusted to get an optimum result in terms of conductivity and roughness. The hot-pressed $\mathrm{Ag}$ NWs/MEH-PPV color conversion electrode with a low sheet resistance of $21.8 \Omega \mathrm{sq}^{-1}$ and a smooth surface is fabricated, which obviously satisfies the requirements of flexible optoelectronic devices. Highly efficient and chromaticity-stable flexible WOLEDs were implemented based on the Ag NWs/MEH-PPV color conversion anode.

\section{Experimental section}

\subsection{General information}

MEH-PPV was purchased from Luminescence Technology Corp (China). Ag NWs solution was purchased from BlueNano Company (Cornelius, NC, USA), with an average diameter of $80 \mathrm{~nm}$ and length of $30 \mu \mathrm{m}$. The other organic functional materials were obtained from e-Ray Optoelectronics Corp. (Taipei, Taiwan). Polyethylene terephthalate (PET) substrates were ordered from CSG Holding Co. Ltd (China). All chemicals and regents in this work were used as received from commercial sources without further treatment unless otherwise stated.

\subsection{Preparation of Ag NWs/MEH-PPV electrode}

The Ag NWs/MEH-PPV color conversion electrodes were prepared by a successive spin-coating process on precleaned
PET substrates. To fabricate the composite film, the PET substrates were cleaned by detergent, de-ionized water, and isopropanol. Ag NWs suspension was spin-coated onto the substrate in air, which was then annealed at $100{ }^{\circ} \mathrm{C}$ for $15 \mathrm{~min}$. Subsequently, the Ag NWs film was hot-pressed by a selfdesigned compressor controlled by compressed air. The pressure intensity applied on the samples can be tuned by adjusting the pressure of compressed air. The Ag NWs film was covered between two clean glass substrates edge to edge, which ensures a smooth and clean contact. And then transferred into the compressor and applied different temperature, pressure and duration to obtain the optimum parameters. Later, MEH-PPV solution was spin-coated on the bottom Ag NWs layer in a glovebox, followed by drying at $60{ }^{\circ} \mathrm{C}$ for $15 \mathrm{~min}$. The thickness of the MEH-PPV CCL was controlled by adjusting the concentration of the solution.

\subsection{Device fabrication}

The substrate coated with Ag NWs/MEH-PPV color conversion electrode was transferred into a high-vacuum system with a base pressure of $5 \times 10^{-6}$ mbar for the following OLED fabrication. The devices structure of the evaporated flexible WOLEDs (Devices W1, W2 and W3) followed as: Ag NWs/MEHPPV $(\lambda \mathrm{nm}) /$ TAPC $(40 \mathrm{~nm}) /$ TCTA $(10 \mathrm{~nm}) / \mathrm{mCP}: 15 \%$ Flrpic $(20$ $\mathrm{nm}) / \mathrm{B} 3 \mathrm{PYMPM}(50 \mathrm{~nm}) / \mathrm{Liq}(0.8 \mathrm{~nm}) / \mathrm{Al}(200 \mathrm{~nm})$, where $\lambda$ are 100, 160 and $200 \mathrm{~nm}$, respectively, as shown in Fig. 1a and b. TCTA $\left(4,4^{\prime}, 4^{\prime \prime}\right.$-tris(carbazol-9-yl)triphenylamine) and TCTA $\left(4,4^{\prime}, 4^{\prime \prime}\right.$-tris(carbazol-9-yl)triphenylamine) serves as hole transporting layer. mCP (1,3-bis(carbazol-9-yl)benzene) is the blue host material. Flrpic (bis(3,5-difluoro-2-(2-pyridyl)phenyl-(2carboxypyridyl)iridium(III))) is the blue phosphorescent guest material. B3PYMPM (4,6-bis(3,5-di(pyridin-3-yl)phenyl)-2methylpyrimidine) is electron transporting layer. Liq (8hydroxyquinolinolato-lithium) and $\mathrm{Al}$ are electron injection layer and cathode, respectively. Chemical structures of the emitting materials described in the OLEDs devices are shown in Fig. 1c. Organic layers and a metal cathode layer were evaporated successively by using shadow masks. The entire organic layers and the $\mathrm{Al}$ cathode were deposited without exposure to the atmosphere, by which OLEDs with an active areas of $4 \mathrm{~mm}^{2}$ were obtained. The deposition rates for the organic materials and Al were typically 1.0 and $5.0 \AA \mathrm{s}^{-1}$, respectively. The layer thickness was controlled in situ using a quartz crystal monitor.

\subsection{Characterization}

The surface morphology of the Ag NWs coated on the PET substrate was investigated by the atomic force microscope (AFM) technique in contact mode using the Seiko instrument SPA 400 AFM system. The sheet resistance is tested with a fourpoint probe. The transmittance of $\mathrm{Ag}$ NWs electrode and absorption spectra of MEH-PPV CCL was measured with an ultraviolet-visible-near infrared spectrophotometer $(\mathrm{U}-3900 \mathrm{H}$, Hitachi). Photoluminescent spectra of MEH-PPV were tested on an Edinburgh Instruments Ltd (FLSP920) fluorescence spectrophotometer in the solid state. The current-voltageluminescence characteristics were measured by a Keithley 2400 
(a)

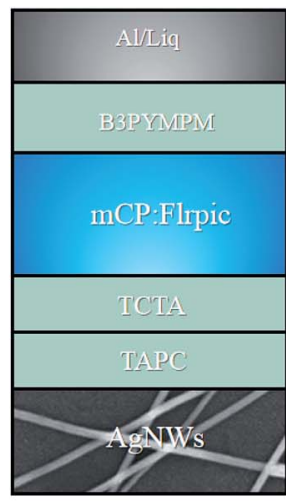

(b)

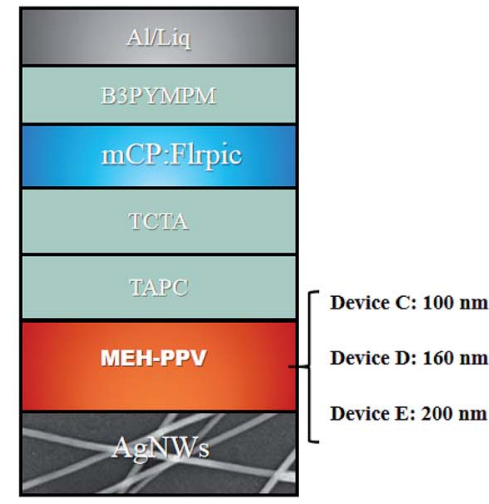

(c)

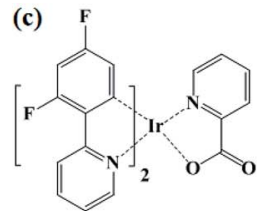

Flrpic

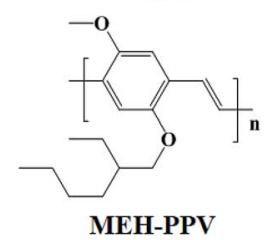

Fig. 1 Schematic structures of the tested flexible (a) blue and (b) white devices with different substrates electrode based on the Ag NWs architecture. (c) Chemical structures of the emitting materials Flrpic and MEH-PPV.

source meter and a PR-650 Spectra Colorimeter. The luminance and spectra of each device were measured in the direction perpendicular to the substrate.

\section{Results and discussion}

\subsection{Optical and electrical properties of $\mathrm{Ag}$ NWs/MEH-PPV composite film}

The transmittance within the visible range and conductivity are two key parameters for transparent conductive electrode. To determine the optimum conditions, the optical transmittance and sheet resistance of $\mathrm{Ag}$ NWs electrode with different suspension concentration $\left(3,4,5,8\right.$ and $\left.10 \mathrm{mg} \mathrm{mL}^{-1}\right)$ are studied and the results are shown in Fig. 2a. When the concentration for $\mathrm{Ag}$ NWs solution increased from $3 \mathrm{mg} \mathrm{mL}^{-1}$ to $10 \mathrm{mg} \mathrm{mL}^{-1}$, the transmittance of the three samples slightly decreased from $89.8 \%$ to $59.5 \%$, respectively. And the change of the sheet resistance shows the similar trend. Here, the figure of merit $\left(\phi_{\mathrm{FOM}}\right)$ is used to characterize the optical and electrical properties of the samples simultaneously, which is defined as follows: ${ }^{25}$

$$
\phi_{\mathrm{FOM}}=\frac{T_{\mathrm{av}}{ }^{10}}{R_{\mathrm{sh}}}
$$

$T_{\mathrm{av}}$ is the average transmittance in the visible light range and $R_{\mathrm{Sh}}$ is the sheet resistance. For the resulting Ag NWs electrode, its figure of merit first increases then decreases with increasing $\mathrm{Ag}$ NWs concentration from $3 \mathrm{mg} \mathrm{mL}{ }^{-1}$. Comparing the $\phi_{\mathrm{FOM}}$ values, $\phi_{\mathrm{FOM}}\left(3 \mathrm{mg} \mathrm{mL}^{-1}\right)=7.12 \times 10^{-3}, \phi_{\mathrm{FOM}}\left(4 \mathrm{mg} \mathrm{mL}^{-1}\right)=$ $7.90 \times 10^{-3}, \phi_{\text {FOM }}\left(5 \mathrm{mg} \mathrm{mL}{ }^{-1}\right)=3.31 \times 10^{-3}, \phi_{\text {FOM }}(8 \mathrm{mg}$ $\left.\mathrm{mL}^{-1}\right)=2.73 \times 10^{-3}$ and $\phi_{\mathrm{FOM}}\left(10 \mathrm{mg} \mathrm{mL}^{-1}\right)=1.71 \times 10^{-3}$. Thus, the Ag NWs film with the concentration of $4 \mathrm{mg} \mathrm{mL} \mathrm{m}^{-1}$ performs the best, which is chosen as the optimum (Fig. 2b).

Mechanical pressure applied on the Ag NWs/MEH-PPV composite film with different temperatures and durations are studied. Here, $R_{\chi}=\left(R-R_{0}\right) / R_{0}$ is used to characterize the changes of $R_{\mathrm{sh}}$ for the Ag NWs/MEH-PPV film during hot pressing process, where $R_{0}$ and $R$ are the sheet resistances before and after hot pressing. ${ }^{26}$ Fig. $3 \mathrm{a}$ and b demonstrates the variation of $R_{\mathrm{sh}}$ and root mean square (RMS) under a pressure from $8 \mathrm{MPa}$ to $50 \mathrm{MPa}$ when the temperature and duration are fixed at $40{ }^{\circ} \mathrm{C}$ and $120 \mathrm{~s}$. At a pressure lower than $13.5 \mathrm{MPa}$, the $R_{\mathrm{sh}}$ changes slightly, indicating that the pressure is too small to flatten the junctions of Ag NWs. However, when the pressure increases to $40 \mathrm{MPa}$, the $R_{\mathrm{sh}}$ changes considerably to $23.6 \Omega$ $\mathrm{sq}^{-1}$ with a diminishment of $35.6 \%$, which proves the pressing can provide a sufficient strength to flatten the junctions and significantly improve the conductivity of Ag NWs/MEH-PPV electrode. Although a slight downtrend is observed in conductivity from $40 \mathrm{MPa}$ to $50 \mathrm{MPa}$, the final $R_{\mathrm{sh}}$ of the films pressed at pressures above $40 \mathrm{MPa}$ are almost the same. However, the $R_{\mathrm{sh}}$
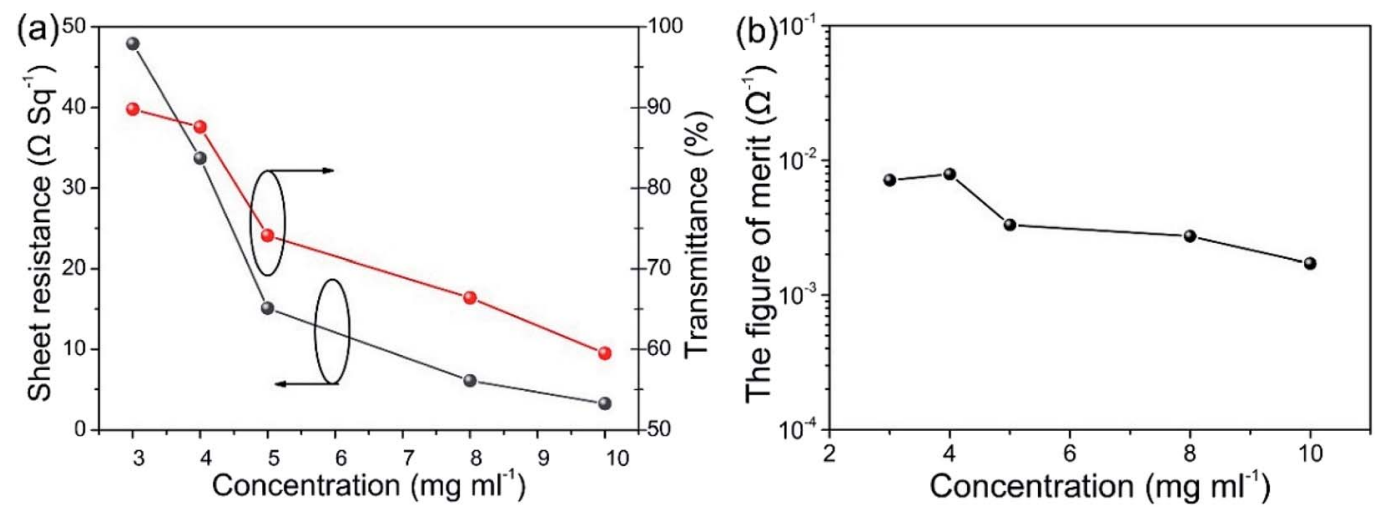

Fig. 2 (a) Sheet resistance (black line) and transmittance (red line) versus the various concentration of Ag NWs suspension and (b) the corresponding figure of merit versus the concentration of Ag NWs suspension. 

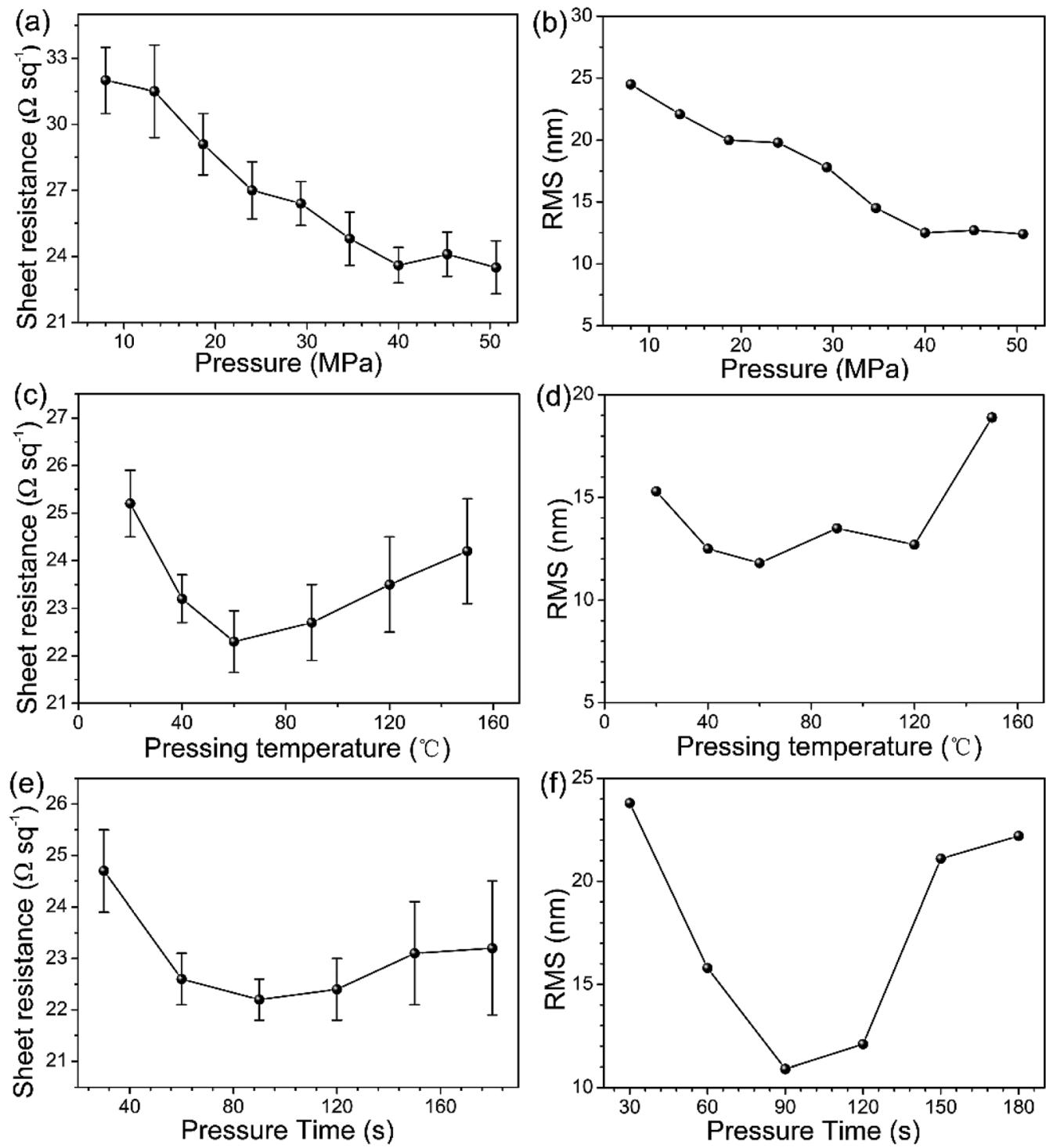

Fig. 3 (a) $R_{\mathrm{sh}}$ versus pressure when temperature and time are $40{ }^{\circ} \mathrm{C}$ and $120 \mathrm{~s}$ and (b) the RMS roughness of the Ag NWs/MEH-PPV composite film versus pressure; (c) $R_{\mathrm{sh}}$ versus pressing temperature when pressure and time are $40 \mathrm{MPa}$ and $120 \mathrm{~s}$ and (d) the RMS roughness of the Ag NWs/ $\mathrm{MEH}-\mathrm{PPV}$ composite film changes; (e) $R_{\mathrm{sh}}$ versus pressing time when pressure and temperature are $40 \mathrm{MPa}$ and $60^{\circ} \mathrm{C}$ and (f) the RMS roughness of the Ag NWs/MEH-PPV composite film changed.

values increase after the press increased from $40 \mathrm{MPa}$ to $50 \mathrm{MPa}$, which can be explained as following: the $R_{\mathrm{sh}}$ is already very small at the press value of $40 \mathrm{MPa}$, suggesting a tight contact between the nanowires and smooth junctions. ${ }^{27}$ Therefore, the hot-pressing may not improve the contacts significantly with press values beyond $40 \mathrm{MPa}$.

Similar phenomenon of $R_{\mathrm{sh}}$ variation is observed when the $\mathrm{Ag}$ NWs/MEH-PPV electrode is annealed with different temperatures under hot pressing process and the pressure and duration are fixed at $40 \mathrm{MPa}$ and $120 \mathrm{~s}$ respectively, as illustrated in Fig. $3 \mathrm{c}$ and $\mathrm{d}$, which shows the variation of $R_{\mathrm{sh}}$ for the $\mathrm{Ag}$ NWs/MEH-PPV film before and after hot-pressing. When the film annealing temperature is as low as $40{ }^{\circ} \mathrm{C}$, the pristine $R_{\mathrm{sh}}$ is high because of the loose contacts for the $\mathrm{Ag} \mathrm{NW}$ junctions, so that hot-pressing can bring a huge decrease of $11.5 \%$ to $R_{\mathrm{sh}}$ by tightly jointing the $\mathrm{Ag}$
NWs. As the annealing temperature directly increase, the contact of nanowires becomes tighter and the effect of pressing is less evident. However, the $R_{\mathrm{sh}}$ has a slight rise when the temperature increases above $60{ }^{\circ} \mathrm{C}$. We speculate the $R_{\text {sh }}$ increase is mainly attributed to poor adhesion between $\mathrm{Ag}$ NWs/MEH-PPV electrode and the substrate at high temperature. ${ }^{28}$ Nevertheless, the final $R_{\mathrm{sh}}$ of films annealed under different temperature are still lower than that of the pristine $R_{\mathrm{sh}}$, which indicates that the conductivity of films with annealed process under hot-pressing have been largely enhanced.

Fig. 3e and $\mathrm{f}$ shows the resistance variations when the pressure duration changes from $30 \mathrm{~s}$ to $180 \mathrm{~s}$, while the pressure intensity and temperature are fixed at $40 \mathrm{MPa}$ and $60^{\circ} \mathrm{C}$. The $R_{\mathrm{sh}}$ decreases from $24.7 \Omega \mathrm{sq}^{-1}$ to $22.2 \Omega \mathrm{sq}^{-1}$ after being hot pressed for $90 \mathrm{~s}$, corresponding to a decline about $10.1 \%$, and possess negligible 
changes when the press time increases from $90 \mathrm{~s}$ to $180 \mathrm{~s}$. As the $R_{\mathrm{sh}}$ at $90 \mathrm{~s}$ is the lowest value among other duration time, we conclude that $90 \mathrm{~s}$ is sufficient to increase the conductivity.

After finishing the preceding optimization studies of $\mathrm{Ag}$ NWs/MEH-PPV, AFM images are employed to further characterize the surface morphologies of the resulting $\mathrm{Ag}$ NWs samples combined with MEH-PPV color conversion layer, as shown in Fig. 4. In the case of Ag NWs/MEH-PPV color conversion electrode before hot-pressing, the RMS is measured at $21.4 \mathrm{~nm}$ (Fig. 4a). In comparison, the RMS of the resulting electrode decreases to $11.8 \mathrm{~nm}$ and the surface is smooth. It is also observed that the surface protuberances are decreasing and the film is getting more and more uniform.

Besides the surface roughness, the flexibility is also a critical parameter for the color conversion electrode. Here, the mechanical flexibility of the composite Ag NWs/MEH-PPV film on PET substrate was evaluated by a bending cycle with a bending radius of $8 \mathrm{~mm}$ under convex and concave compression, and the sheet resistance of the sample is measured every 25 bending cycles. $R_{0}$ and $R$ represent the sheet resistance before and after bending, respectively. An ITO electrode fabricated on PET substrate was also tested as reference. As shown in Fig. S1, $\uparrow$ during the bending test, the $R_{\text {sh }}$ of the ITO electrode increases dramatically and the sheet resistance elevates two orders of magnitude after 600 bending cycles. In contrast, the $R_{\text {sh }}$ of the hot-pressed Ag NWs/MEH-PPV color conversion electrode does not alter significantly and keeps almost unchanged during the bending test, which indicates the presence of the tight connections between the Ag NWs. ${ }^{29}$

\subsection{Performance of the flexible blue phosphorescent OLED}

To verify the performance of the hot pressed Ag NWs/MEH-PPV with low resistance and surface roughness and evaluate the potential for practical applications, we firstly fabricated flexible blue phosphorescent OLEDs using the Ag NWs as anodes. Fig. 1a schematically displays the structure of the Ag NWs based blue OLEDs on a flexible PET substrate and the electroluminescent performance of the flexible blue OLED is shown in Fig. 5 and Table 1. The blue OLED shows a maximum luminance of $9585.6 \mathrm{~cd} \mathrm{~m}^{-2}$ and a low turn-on voltage $\left(V_{\text {on }}\right)$ of $3.8 \mathrm{~V}$, where the $V_{\text {on }}$ are extracted at the luminance value of $1 \mathrm{~cd} \mathrm{~m}^{-2}$ (Fig. 5a). This low $V_{\text {on }}$ can be attributed to the high optoelectronic performance of our Ag NWs electrode, the thick junctions on the surface of the hot-pressed Ag NWs film, which are prone to form current pathways between the electrodes. Current efficiency (CE) and external quantum efficiency (EQE) versus luminance are shown in Fig. $5 \mathrm{~b}$ and $\mathrm{c}$. The maximum $\mathrm{CE}$ of the device with $\mathrm{Ag}$ NWs anode is $16.3 \mathrm{~cd} \mathrm{~A}^{-1}$, which is one of the best values among reported Ag NWs anode-based OLEDs in the literature. ${ }^{\mathbf{3 0 , 3 1}}$ Furthermore, the Ag NWs based OLED shows an excellent external quantum efficiency of over $8.5 \%$ with the maximum emission peak at $468 \mathrm{~nm}$ (Fig. 5d).

Fig. S2 $\uparrow$ shows the absorption and photoluminescence spectra of Ag NWs/MEH-PPV color conversion electrode and the EL spectra of Flrpic. The absorption spectrum has a maximum peak at $489 \mathrm{~nm}$. The EL spectrum of emitting layer Flrpic overlaps well with the absorption spectrum of the composite electrode. We expect the efficient down-conversion from blue photons to longer wavelength yellow photons and realize the (a)
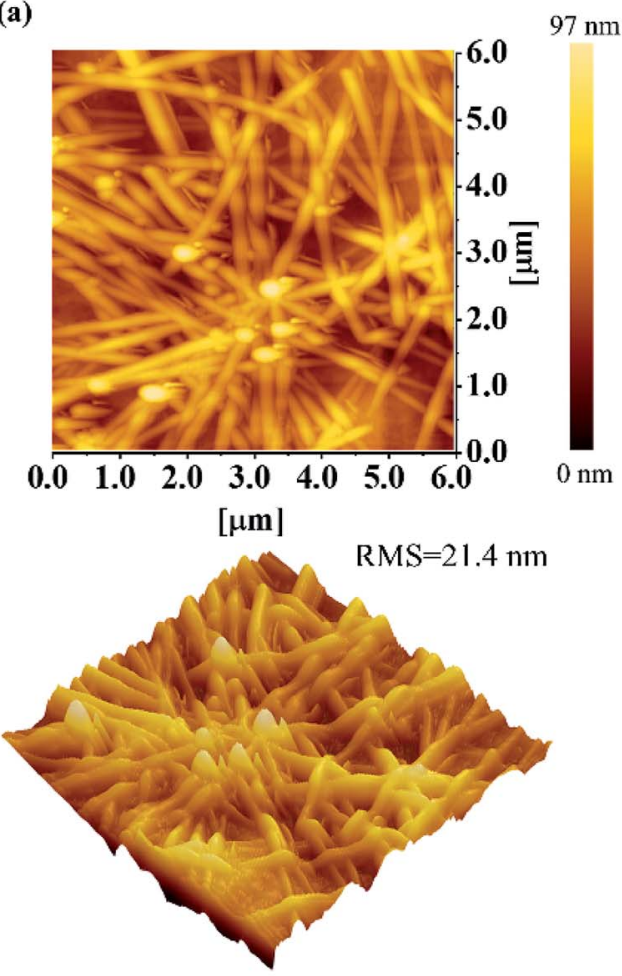

(b)
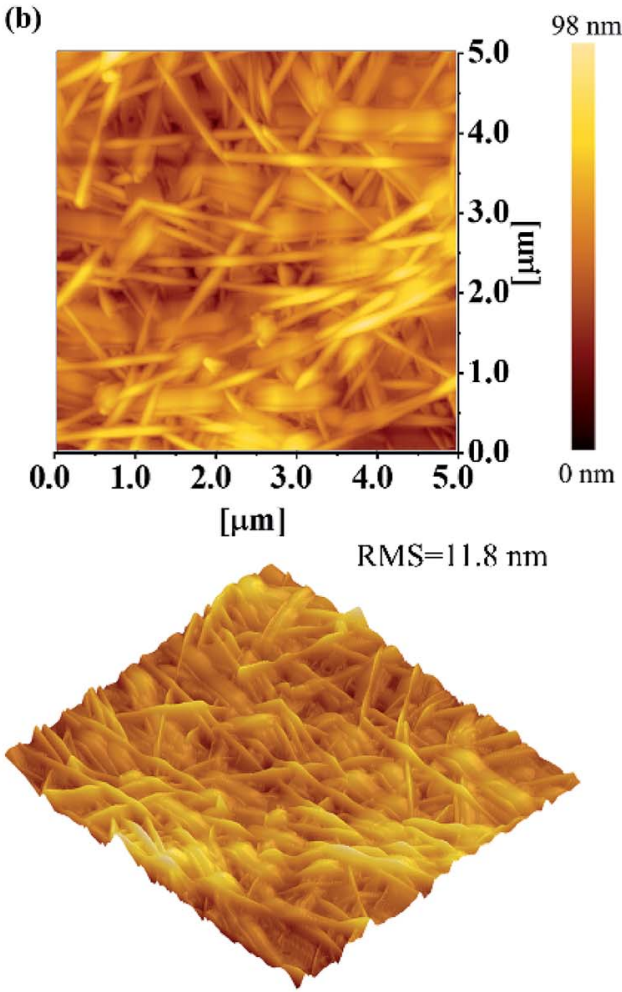

Fig. 4 AFM images of optimized Ag NWs/MEH-PPV composite film on PET substrate (a) before and (b) after hot-pressing at $40 \mathrm{MPa}$ and $60{ }^{\circ} \mathrm{C}$ for $90 \mathrm{~s}$. 

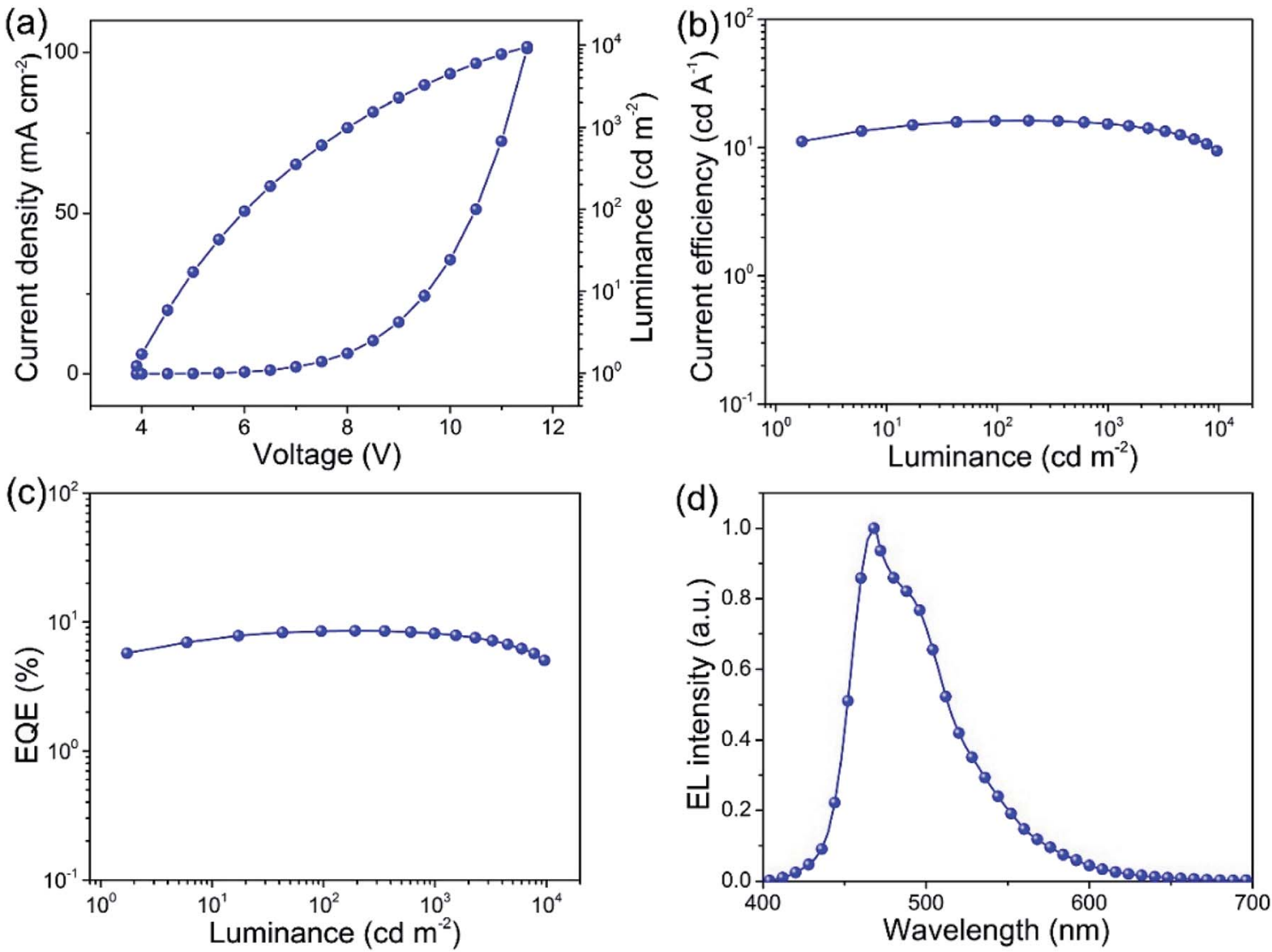

Fig. 5 Performance of phosphorescent blue OLEDs. (a) Current density-luminance-voltage (J-V-L) characteristics; (b) current efficiency versus luminance; (c) external quantum efficiency versus luminance and (d) EL emission spectra.

WOLEDs. The yellow emission from the excited MEH-PPV CCL was centered at $586 \mathrm{~nm}$. The extent of color conversion can be controlled by the amount of the CCL, and the correlated color temperature of flexible WOLEDs is determined by an appropriate balance between the converted yellow emission and the blue EL emission which has not been converted..$^{32}$ The amount of the MEH-PPV CCL is precisely controlled in order to achieve a high performance white emission. To achieve this goal easily, we prepared a single MEH-PPV CCL and the thickness of CCL was adjusted by controlling the concentration of MEH-PPV layer.

\subsection{Performance of the flexible WOLEDs}

With the optimization of the fabrication process of blue OLEDs based on Ag NWs electrode, we fabricate flexible WOLEDs as a targeted application and to simplify the WOLEDs fabrication where the Ag NWs-based electrode combined with MEH-PPV acts as a color conversion anode of the WOLEDs with various thickness of MEH-PPV CCL (Device W1: $100 \mathrm{~nm}$, Device W2: $160 \mathrm{~nm}$ and Device W3: $200 \mathrm{~nm}$ ). The WOLEDs device structure is shown in Fig. 1b. Fig. 6 summaries the current density $(J)$ versus voltage, luminance versus voltage, current and external quantum efficiencies versus luminance $(L)$ and the emission spectra of the resulting flexible WOLEDs.

The Ag NWs/MEH-PPV-based WOLEDs (Device W1 and W2) reveal a sharp increase of $J$ values and the low turn-on voltages of $4.2 \mathrm{~V}$ and $4.3 \mathrm{~V}$, respectively. The maximum $J$ values of the three WOLEDs with various thickness of MEH-PPV CCL (100, 160 and $200 \mathrm{~nm}$ ) are $100.5,116.8$, and $49.0 \mathrm{~mA} \mathrm{~cm}^{-2}$, respectively, with the corresponding maximum $L$ values of 11982.0 , 7079.1 and $2723.0 \mathrm{~cd} \mathrm{~m}^{-2}$ (Fig. 6a and b). Moreover, the peak

Table 1 Summary of flexible OLEDs characteristics using various Ag NWs architecture

\begin{tabular}{|c|c|c|c|c|c|c|}
\hline Device $^{a}$ & Anode & $V_{\text {on }}^{b}[\mathrm{~V}]$ & $L^{c}\left[\mathrm{~cd} \mathrm{~m}^{-2}\right]$ & $\mathrm{CE}^{d}\left[\mathrm{~cd} \mathrm{~A}^{-1}\right]$ & $\mathrm{EQE}^{e}[\%]$ & $\operatorname{CIE}^{f}[x, y]$ \\
\hline B & $\mathrm{Ag}$ NWs & 3.8 & 9585.6 & 16.3 & 8.5 & $0.17,0.39$ \\
\hline W1 & Ag NWs/MEH-PPV (100 nm) & 4.2 & 11982.0 & 20.5 & 9.1 & $0.28,0.30$ \\
\hline W3 & Ag NWs/MEH-PPV (200 nm) & 5.5 & 2737.0 & 7.2 & 2.7 & $0.43,0.41$ \\
\hline
\end{tabular}

${ }^{a}$ Device configuration: anode/TAPC $(40 \mathrm{~nm}) /$ TCTA $(10 \mathrm{~nm}) / \mathrm{mCP}: 15 \%$ Flrpic $(20 \mathrm{~nm}) /$ B3PYMPM $(40 \mathrm{~nm}) / \mathrm{Liq}(1 \mathrm{~nm}) / \mathrm{Al}(100 \mathrm{~nm}) .{ }^{b}$ The operating voltage at a brightness of $1 \mathrm{~cd} \mathrm{~m}^{-2} .{ }^{c} L$, maximum luminance. ${ }^{d} \mathrm{CE}$, maximum current efficiency. ${ }^{e} \mathrm{EQE}$, maximum external quantum efficiency.

${ }^{f}$ CIE coordinates. 

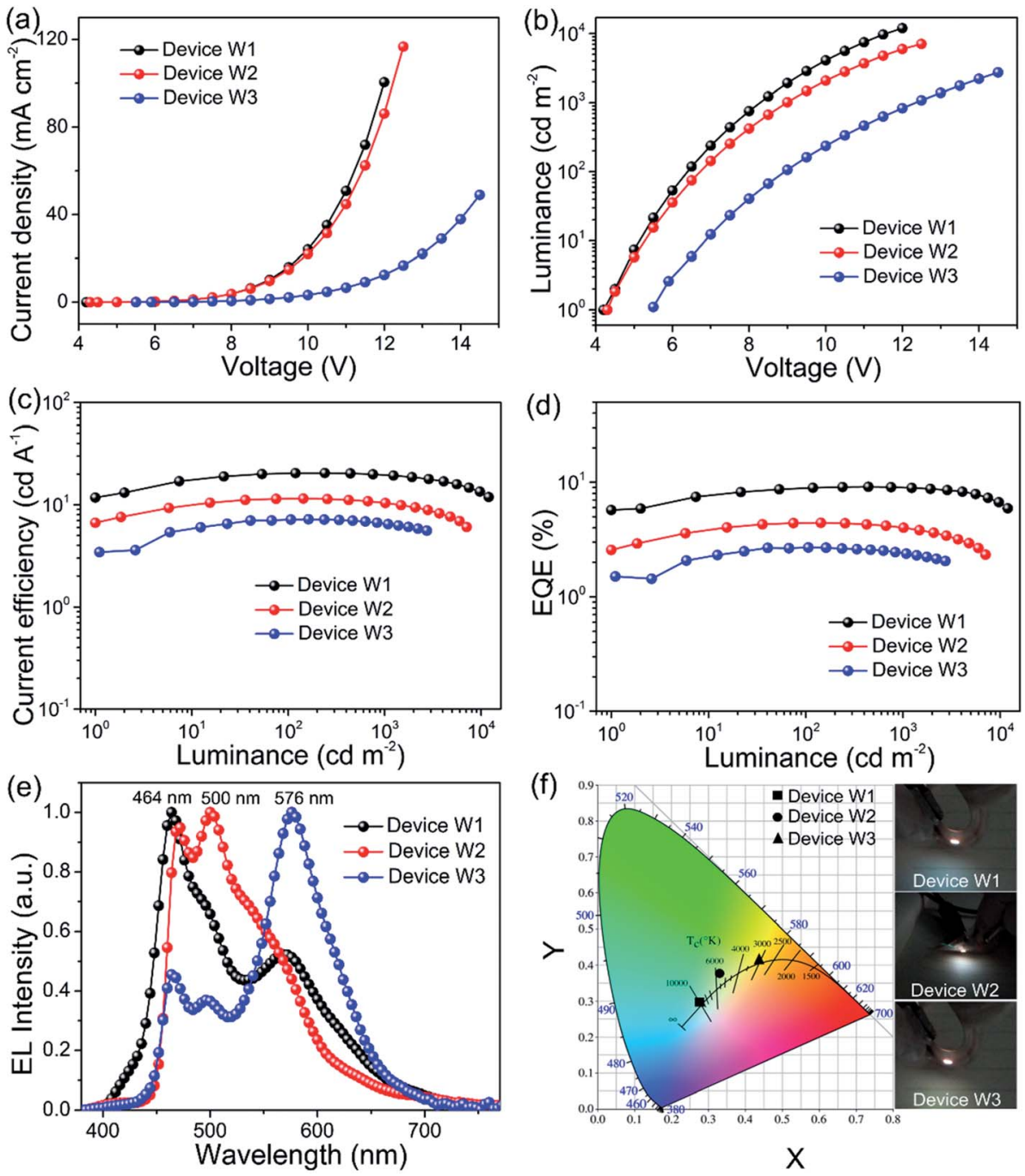

Fig. 6 Performance of flexible WOLEDs fabricated on PET substrates based on Ag NWs/MEH-PPV color conversion anode. (a) Current density versus voltage (b) luminance versus voltage (c) CE versus luminance, (d) EQE versus luminance, (e) electroluminescent spectra, (f) the corresponding Commission Internationale de L'Eclairage (CIE) coordinates. (Inset: photographs of the flexible WOLEDs.)

CEs and EQEs of the three WOLEDs are extracted from the measured $J-V-L$ properties as shown in Fig. $6 \mathrm{c}$ and d, to further investigate the effect of different thicknesses of MEH-PPV CCL. The maximum CE of the device W1 with $100 \mathrm{~nm} \mathrm{MEH-PPV}$ is $20.5 \mathrm{~cd} \mathrm{~A}^{-1}$ with the corresponding CIE coordinates of $(0.28$, 0.30 ), representing one of best performances compared to previously reported CCL based flexible WOLEDs. ${ }^{33}$ A comparison between our result and other CCL-based WOLEDs is presented in Table S1. $\dagger$ When increasing the thickness of $\mathrm{MEH}-$ PPV layers to $160 \mathrm{~nm}$ and $200 \mathrm{~nm}$, the maximum CE gradually decreases to 11.5 and $7.2 \mathrm{~cd} \mathrm{~A}^{-1}$, respectively. This tendency is reconfirmed by the reduction of the maximum $\mathrm{EQE}$ in the flexible WOLEDs, where EQEs of $9.1 \%, 4.4 \%$ and $2.7 \%$ are observed for the device $\mathrm{W} 1$, device $\mathrm{W} 2$ and device $\mathrm{W} 3$, respectively. The reason of this tendency might be attributed to the decrease of optical transmittance and conductivity, which strongly affects the performance of the WOLEDs. Fig. 6e shows the EL spectra of the three tested WOLEDs with different thicknesses of CCL. Device W1 exhibited cold white emission, indicating insufficient energy transfer from Flrpic to MEH-PPV CCL. Further increasing the thickness of CCL to $160 \mathrm{~nm}$, as predicted, device W2 showed standard white EL spectra, and the CIE coordinate was located at $(0.33,0.37)$ (Fig. 6f). The emission peak of Device W3 is shown at a wavelength of $576 \mathrm{~nm}$, which is mainly generated from the thicker MEH-PPV CCL in comparison to the main emission peaks (464 nm and $500 \mathrm{~nm}$ ) from Flrpic for Devices W1 and W2.

To further examine the mechanical stability of the resulting WOLEDs, the device W1 with the highest efficiency was selected as an example to illustrate this point. The temporal change in luminance was monitored under bending deformations in glove box. As shown in Fig. S3, $\uparrow$ the device W1 exhibit excellent mechanical flexibility, the initial brightness of $2000 \mathrm{~cd} \mathrm{~m}^{-2}$ can be maintained over $81 \%$ with bending radius of $8 \mathrm{~mm}$ under convex and concave compression for 600 cycles. The good stability can be attributed to the good mechanical compliant property of Ag NWs/MEH-PPV, efficient charge carrier injection and high electrical conductivity of the electrodes. Considering 


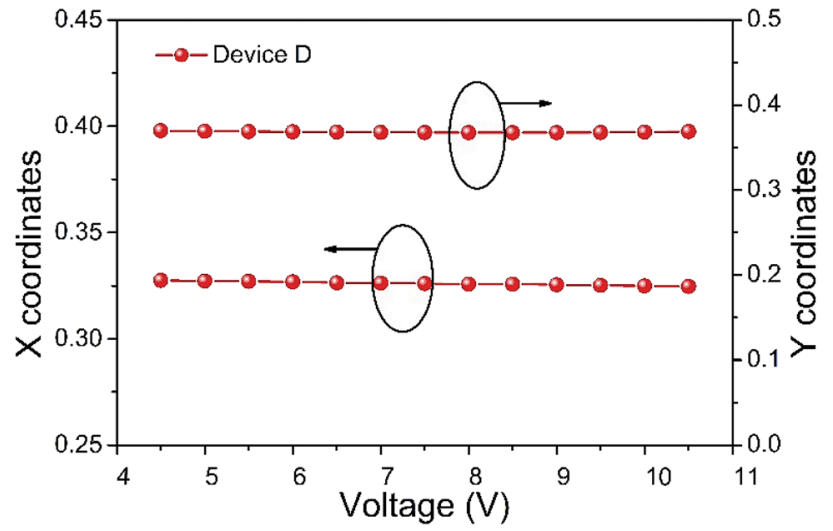

Fig. 7 Shifts in CIE $1931 X Y$-coordinates for the device W2 according to the working voltage changes.

that insignificant change in the $R_{\mathrm{S}}$ of the $\mathrm{Ag}$ NWs/MEH-PPV electrode was observed at this level of bending radius, the slight decrease in luminance may result from the degradation of the stiffer interfacial layers, such as the vacuum-evaporated $\mathrm{Al}$ or interfacial contacts by mechanical deformation. These results suggest that combining WOLEDs with Ag NWs/MEHPPV color conversion electrode enables the fabrication of extremely flexible and high-performance solid-state lightings.

CIE color coordinates of the device W2 under study is shown in $0.5 \mathrm{~V}$ steps of driving voltage in Fig. 7. The $X$-coordinate of the WOLED varies from $0.3276(4.5 \mathrm{~V}$ ) to 0.3261 (at 7.5 V) and 0.3247 (at $10.5 \mathrm{~V}$ ). This corresponds to a relatively 'standard white' color with a correlated color temperature around 5000-6000 K. Differences of the coordinates were negligible due to the intrinsic color stability advantage of the combination of a single-emitter OLED and a color-conversion layer.

\section{Conclusions}

In conclusion, a highly flexible, conductive and smooth Ag NWs/MEH-PPV color conversion electrode has been fabricated by a simple hot-pressing method. The solution-processed $\mathrm{Ag}$ NWs/MEH-PPV composite film shows a low sheet resistance of $21.8 \Omega \mathrm{sq}^{-1}$ and a low surface roughness. Using the Ag NWs/ MEH-PPV composite film as the anode, superior performance of the down-conversion flexible WOLEDs with a maximum current efficiency of $20.5 \mathrm{~cd} \mathrm{~A}^{-1}$ is achieved with the corresponding CIE coordinates of $(0.28,0.30)$, which is the first report for the color-conversion electrode based on the Ag NWs for flexible WOLEDs devices. Our work also provides a new avenue of achieving highly efficient flexible optoelectronics for solid-state lighting and display applications.

\section{Author's contributions}

C. P. and K. L. conceived the new idea and strategy, designed and fabricated the devices, and performed the measurements and analysis with assistance from Z. T., Z. L., H. L., G. C., Y. L., and W. L. B. W. supervised this project and prepared the manuscript with assistance from the other co-authors S. W. and N. Y. All authors have approved the final version of the manuscript.

\section{Conflicts of interest}

The authors declare no competing financial interests.

\section{Acknowledgements}

This work is financially supported by the National Key Research and Development Program of China (No. 2016YFB0401303).

\section{References}

1 Y. Miao, K. Wang, B. Zhao, L. Gao, P. Tao, X. Liu, Y. Hao, H. Wang, B. Xu and F. Zhu, Nanophotonics, 2018, 7, 295-304. 2 S. Wang, M. Qiao, Z. Ye, D. Dou, M. Chen, Y. Peng, Y. Shi, X. Yang, L. Cui, J. Li, C. Li, B. Wei and W. Y. Wong, iScience, 2018, 9, 532-541.

3 G. Shi, X. Zhang, M. Wan, S. Wang, H. Lian, R. Xu and W. Zhu, RSC Adv., 2019, 9, 6042-6047.

4 Y. Wu, J. Yang, S. Wang, Z. Ling, H. Zhang and B. Wei, Appl. Sci., 2019, 9, 1415.

5 S. Wang, L. Zhao, B. Zhang, J. Ding, Z. Xie, L. Wang and W. Y. Wong, iScience, 2018, 6, 128-137.

6 T. Xu, J.-G. Zhou, M.-K. Fung and H. Meng, Org. Electron., 2018, 63, 369-375.

7 Z. Wu and D. Ma, Mater. Sci. Eng., R, 2016, 107, 1-42.

8 D. Luo, C. T. Hsieh, Y.-P. Wang, T. C. Chuang, H.-H. Chang and C. H. Chang, RSC Adv., 2018, 8, 30582-30588.

9 C. H. Chang, Y. J. Lo, J. L. Huang, Y. F. Huang, H. Y. Hung, Y. F. Jang and H. H. Chang, Org. Electron., 2014, 15, 19061912.

10 S. Chen and H.-S. Kwok, Org. Electron., 2011, 12, 677-681.

11 L. Tang, S. Cheng, L. Zhang, H. Mi, L. Mou, S. Yang, Z. Huang, X. Shi and X. Jiang, iScience, 2018, 4, 302-311.

12 J. Lewis, S. Grego, B. Chalamala, E. Vick and D. Temple, Appl. Phys. Lett., 2004, 85, 3450-3452.

13 C. Guillén and J. Herrero, Thin Solid Films, 2011, 520, 1-17. 14 X. Crispin, F. L. E. Jakobsson, A. Crispin, P. C. M. Grim, P. Andersson, A. Volodin, C. V. Haesendonck, M. V. D. Auweraer, W. R. Salaneck and M. Berggren, Chem. Mater., 2006, 18, 4354-4360.

15 D. Langley, G. Giusti, C. Mayousse, C. Celle, D. Bellet and J. P. Simonato, Nanotechnology, 2013, 24, 452001.

16 Y. S. Oh, H. Choi, J. Lee, H. Lee, D. Y. Choi, S. U. Lee, K. S. Yun, S. Yoo, T. S. Kim, I. Park and H. J. Sung, Sci. Rep., 2017, 7, 11220.

17 S. Wang, J. Yang, T. Xu, D. Dou, Z. Tang, Z. Gao, M. Chen, K. Guo, J. Yu, J. Plain, R. Bachelot, J. Zhang and B. Wei, Org. Electron., 2019, 64, 146-153.

18 S. Coskun, E. Selen Ates and H. E. Unalan, Nanotechnology, 2013, 24, 125202.

19 S. Wang, Y. Zhao, H. Lian, C. Peng, X. Yang, Y. Gao, Y. Peng, W. Lan, O. I. Elmi, D. Stiévenard, B. Wei, F. Zhu and T. Xu, Nanophotonics, 2019, 8, 297-306. 
20 S. Kim, H. Lee, S. Na, E. Jung, J. G. Kang, D. Kim, S. M. Cho, H. Chae, H. K. Chung, S. B. Kim, B. W. Lee, K. E. Kim, S. Lee, H. J. Lee, H. Kim and H. J. Lee, Nanotechnology, 2015, 26, 135705.

21 W. Gaynor, S. Hofmann, M. G. Christoforo, C. Sachse, S. Mehra, A. Salleo, M. D. McGehee, M. C. Gather, B. Lussem, L. Muller-Meskamp, P. Peumans and K. Leo, Adv. Mater., 2013, 25, 4006-4013.

22 L. Lian, D. Dong, D. Feng and G. He, Org. Electron., 2017, 49, 9-18.

23 Y.-S. Kim, E.-J. Lee, J.-T. Lee, D.-K. Hwang, W.-K. Choi and J.-Y. Kim, RSC Adv., 2016, 6, 64428-64433.

24 K. Zilberberg, F. Gasse, R. Pagui, A. Polywka, A. Behrendt, S. Trost, R. Heiderhoff, P. Görrn and T. Riedl, Adv. Funct. Mater., 2014, 24, 1671-1678.

25 G. Haacke, J. Appl. Phys., 1976, 47, 4086-4089.
26 E. Jung, C. Kim, M. Kim, H. Chae, J. H. Cho and S. M. Cho, Org. Electron., 2017, 41, 190-197.

27 B. Wei, X. Wu, L. Lian, S. Yang, D. Dong, D. Feng and G. He, Org. Electron., 2017, 43, 182-188.

28 Y. Jin, D. Deng, Y. Cheng, L. Kong and F. Xiao, Nanoscale, 2014, 6, 4812-4818.

29 M. Hu, J. Gao, Y. Dong, K. Li, G. Shan, S. Yang and R. K. Li, Langmuir, 2012, 28, 7101-7106.

30 W. Zhou, J. Chen, J. Chen, C. Zheng, Z. Gao, B. Mi, H. Zhang and Y. Ma, J. Nanosci. Nanotechnol., 2016, 16, 12609-12616.

31 E. Jung, C. Kim, M. Kim, H. Chae, J. H. Cho and S. M. Cho, Org. Electron., 2017, 41, 190-197.

32 T. W. Koh, H. Cho, C. Yun and S. Yoo, Org. Electron., 2012, 13, 3145-3153.

33 B. S. Kim, M. K. Kim, D. S. Jo, H. Chae and S. M. Cho, ECS J. Solid State Sci. Technol., 2018, 7, 3176-3181. 